\title{
AN EXPERIMENTAL STUDY OF EXTREME WAVE KINEMATICS ON OPPOSING DEPTH-VARYING CURRENTS
}

\author{
Xuyang Niu, Dalian University of Technology, niuxuyang@mail.dlut.edu.cn \\ Yuxiang Ma, Dalian University of Technology, yuxma@dlut.edu.cn \\ Xiaozhou Ma, Dalian University of Technology, maxzh@dlut.edu.cn \\ Guohai Dong, Dalian University of Technology, ghdong@dlut.edu.cn \\ Yongbo Song, Guangdong Province Planning and Design Institute for Water Transportation, 847395624@qq.com
}

\section{INTRODUCTION}

In the past decades, there are numerous reports on the accidents caused by extremely large waves. A large number of papers have contributed to the study on the physical properties and the generation mechanisms of such large waves. However, most of the research has been carried out without presence of currents. In the real ocean, the waves most likely coexist with currents. In many circumstances, owing to wind force and oceanic circulation, the currents are commonly not uniform and vary with depth, which plays an important role in the evolution of gravity waves (Swan et al., 2001). Wave kinematics is a key factor in designing structures. However, experimental studies of the influence of depth-varying currents on extreme waves are mainly considered for the changing of spatial and temporal geometric characteristics (Yao and $\mathrm{Wu}, 2006$ ). In the present study, the kinematics of extreme waves on opposing depth-varying currents will be investigated using the PIV technology.

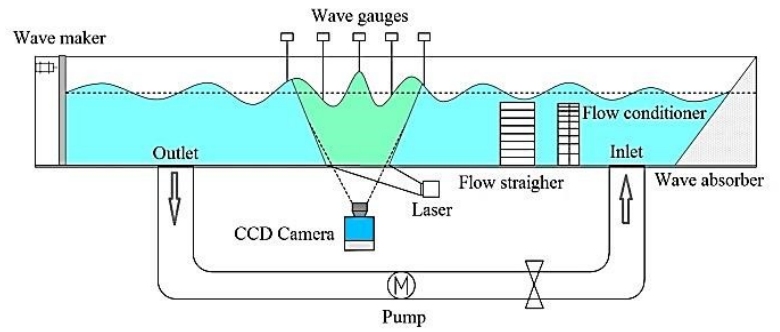

Figure 1 -Schematic sketch of the experimental set-up

Table 1 Wave and current parameters

\begin{tabular}{ccccc}
\hline Case & $f(\mathrm{~Hz})$ & $A(\mathrm{~cm})$ & $\begin{array}{c}\text { Surface current } \\
\text { velocity }(\mathrm{m} / \mathrm{s})\end{array}$ & $\begin{array}{c}\text { Current } \\
\text { Shear }\left(\mathrm{s}^{-1}\right)\end{array}$ \\
\hline A3 & & 3 & 0 & 0 \\
A3U9 & & 3 & -0.09 & 0 \\
A3US9 & $0.8-1.2$ & 3 & -0.09 & 0.225 \\
A5 & 5 & 0 & 0 \\
A5U9 & 5 & -0.09 & 0 \\
A5US9 & 5 & -0.09 & 0.225 \\
\hline
\end{tabular}

\section{EXPERIMENTAL SETUP}

The experiment was conducted in a wave-current flume located at the State Key Laboratory of Coastal and Offshore Engineering, Dalian University of Technology. The flume is $20 \mathrm{~m}$ long, $0.45 \mathrm{~m}$ wide, and filled with a water depth of $d=0.4 \mathrm{~m}$. The detailed setup is shown in Figure 1 . The currents were generated by a pump with a closed-loop pipeline. A honeycomb block was placed downstream the flow inlet to straighten the flow velocity. To generate controllable depth-varying shear currents in the flume, a flow conditioner apparatus was installed (see Figure 1). The velocity and vorticity of currents were controlled by the porosity of the honeycomb blocks. The measured velocity profiles of the uniform and shear currents were depicted in Figure 2, where $U_{s}$ and $U^{\prime}$ are surface current velocity and current vorticity. In the present study, extreme waves based on JONSWAP spectra were generated by the dispersive focusing method (see Table 1). The PIV technique was employed to obtain the velocity field under extreme crests.

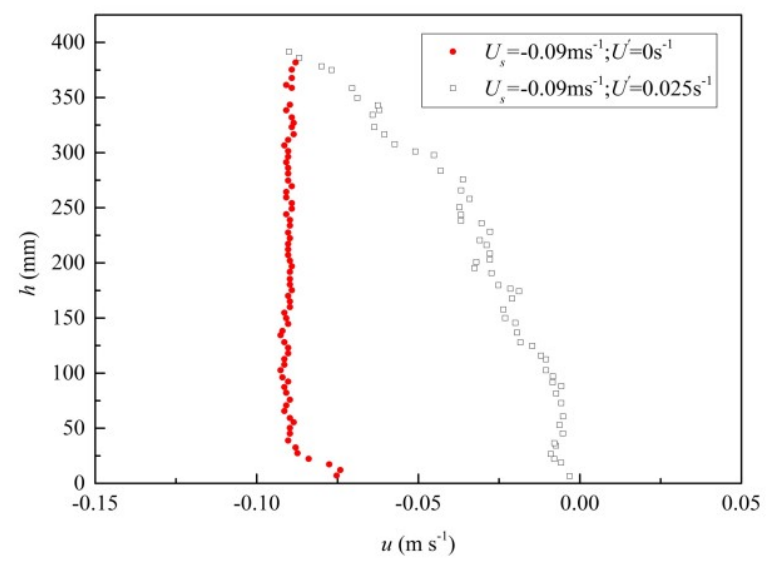

Figure 2 -Vertical velocity profiles of the currents

\section{RESULTS AND DISCUSSION}

Herein, the fifth-order Stokes theory is adopted to depict the distribution of extreme wave kinematic without current $(U=0)$, and the expressions are omitted here and referred to Fenton (1985). Because the extreme waves are multi-chromatic and exhibit signs of transience, the local characteristic parameters (height and period) are extracted to describe the velocity distribution. Three different definitions of the characteristic wave for the extreme waves are outlined in Table 2. The meanings of parameters are depicted in Figure 3. Furthermore, the measured vertical velocity profile under the extreme crest (case A3) are presented in figure 4 , where the calculated solutions with various parameters outlined in Table 2 are also embedded for comparison.

Table 2 Characteristic parameters

\begin{tabular}{ccc}
\hline Model & Height & Period \\
\hline $\mathrm{A}$ & $H_{a}=H_{1}$ & $T_{a}=T_{1}$ \\
$\mathrm{~B}$ & $H_{b}=H_{1}$ & $T_{b}=2 T_{2}$ \\
$\mathrm{C}$ & $H_{c}=2 H_{2}$ & $T_{c}=2 T_{3}$ \\
\hline
\end{tabular}

The velocity profile shows hyperbolic cosine distribution along the water depth. Definitely, the velocity distribution that based on the model $C$ was significantly overestimated. As for the model $A$, the predicted velocities are overestimated in the part beneath the MWL ( $h=400 \mathrm{~mm})$ against the measured results. Furthermore, the variation of horizontal velocities in the depth is slower than the measured one, and therefore the velocities are slightly underestimated for the upper part. The model B can fit better with the experimental data along the whole depth, indicating that the $5^{\text {th }}$-order 
Stokes kinematic velocity based on the model B might be an effective way in description of the extreme wave kinematics.

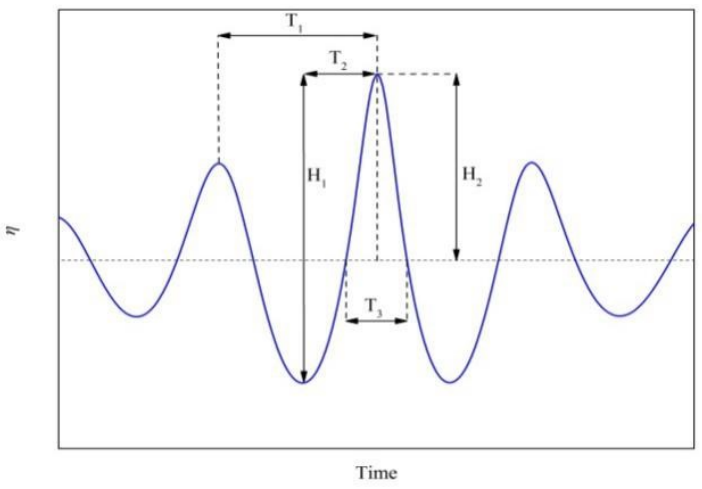

Figure 3 -Definition of characteristic parameters

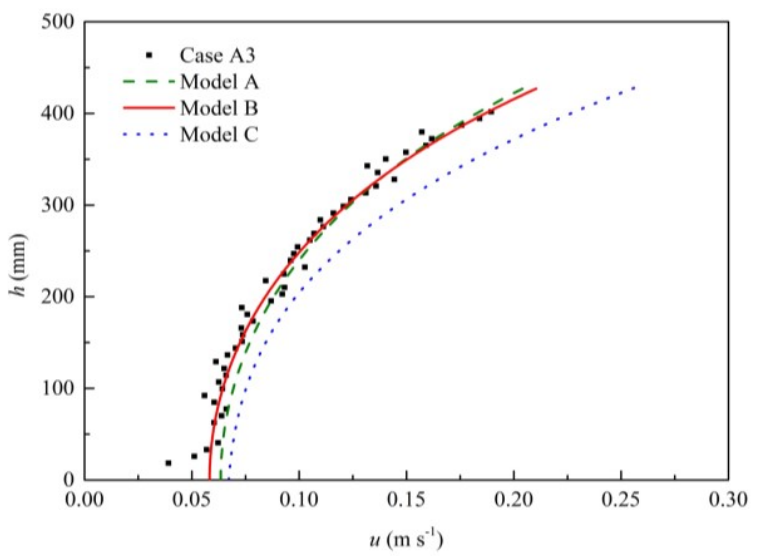

Figure 4 -Horizontal velocities under extreme wave crests for case $A 3$

With the input focusing amplitude of $3 \mathrm{~cm}$, the measured horizontal velocity profiles under the maximum crest of all cases are depicted in Figure 5. Additionally, the Dopplershifted solutions on the uniform and the shear currents are presented for comparison. It should be noted that such solutions are the subtraction from the $5^{\text {th }}$-order Stokes prediction (based on model B) with the measured currents shown in Figure 2. On the effect of depthvarying current, the measured velocity profile is shifted from the solution in quiescent water. For the part farther away from MWL $(0<h / d<0.8)$, the velocity changes slow along the depth and fits well with the Doppler-shifted solution. However, in the area near the wave surface $(h / d>0.8)$, the change rate of horizontal velocity is relatively large and the Doppler-shifted solution underestimates the velocity significantly. Regarding the velocities on the opposing uniform current, the measured profile fits well with Doppler-shifted solution, but the deviation occurs from $h / d>0.76$. Meanwhile, for the part upon MWL the velocities are coincident with the ones on the opposing shear current. Similar results were obtained with larger input amplitude.

The present experimental results indicate that the influence of shear currents on wave kinematics is far more than linear correction. In the lower part beneath MWL, the effects of opposing uniform and shear current are linear and obey the rules of Doppler shift. However, in the area close to the wave surface, the nonlinear interaction is severer and Doppler-shift predictions tend to underestimate the horizontal velocities.
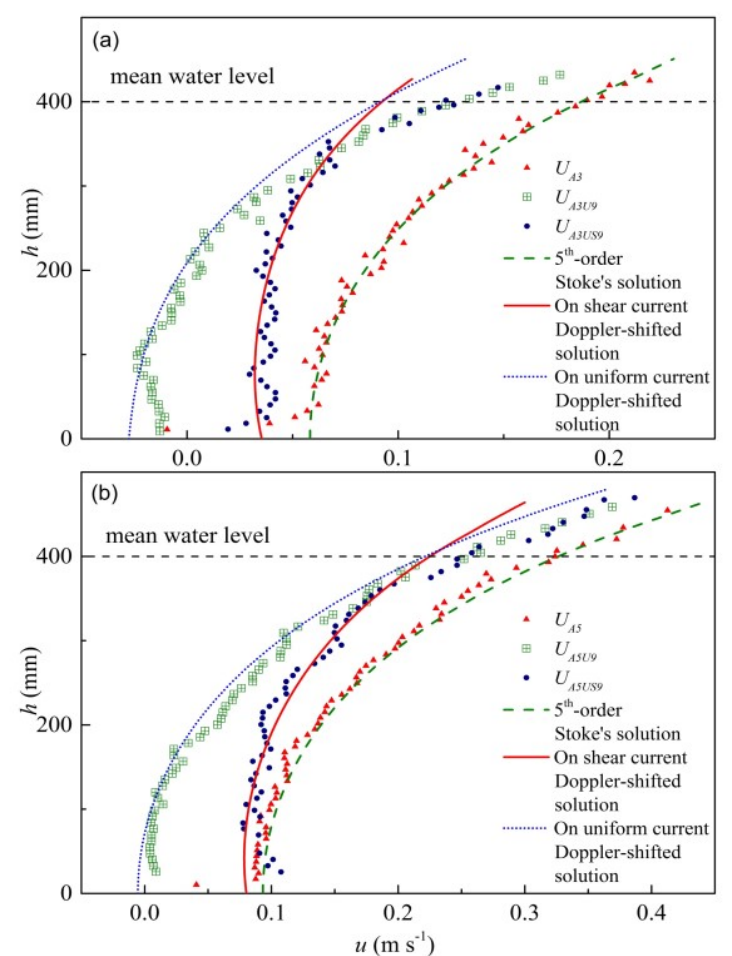

Figure 5 -Horizontal velocities under extreme wave crests, (a) case A3, A3U9 and A3US9; (b) case A5, A5U9 and A5US9

\section{CONCLUSION}

Experiment of extreme wave kinematics on opposing depth-varying currents has been carried out. An improved model based on the $5^{\text {th }}$-order of Stokes solution was proposed to predict the distribution of extreme wave kinematic. The characteristic height of such model is defined as the amplitude difference of focal crest and adjacent tough, and the characteristic period is twice the time span of focal crest and adjacent crest. As a result, the $5^{\text {th }}$-order Stokes kinematic velocities based on such model are coincident well with the measured ones. Under the effects of the opposing uniform and the shear currents, lower part of horizontal profile fits well with the Doppler-shifted 5th-order solution. However, in the area near the wave surface, the horizontal velocity changes fast and the Dopplershifted solution underestimates the measured velocity significantly.

\section{ACKNOWLEDGEMENTS}

This research is supported financially by the High-Tech Ship Research Projects Sponsored by the Ministry of Industry and Information Technology (MIIT) of China.

\section{REFERENCES}

Fenton, J. D. (1985): A fifth-order stokes theory for steady waves. Journal of Waterway Port Coastal \& Ocean Engineering, 111(2), 216-234.

Swan, C., Cummins, I.P. and James, R.L. (2001): An experimental study of two-dimensional surface water waves propagating on depth-varying currents. Part 1. Regular waves. Journal of Fluid Mechanics, 428, 273304.

Yao, A. and Wu, C.H. (2006): Spatial and temporal characteristics of transient extreme wave profiles on depth-varying currents. Journal of Engineering Mechanics, 132(9), 1015-1025. 